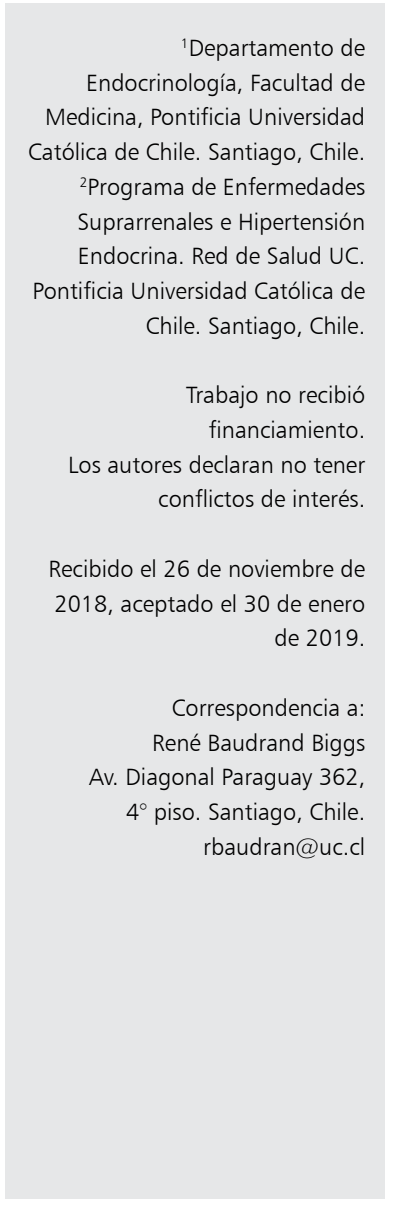

\section{Actualización en el manejo clínico de la hipertensión hiporreninémica}

\author{
STEFANO MACCHIAVELLO ${ }^{1}$, CARLOS FARDELLA ${ }^{1,2}$, \\ RENÉ BAUDRAND ${ }^{1,2}$

\section{Update in the clinical management of low renin hypertension}

The renin-angiotensin-aldosterone system modulates volume, sodium and potassium homeostasis. In the setting of a high sodium diet, up to $30 \%$ of patients with hypertension have a low or suppressed renin and increased volume. This phenotype of low renin hypertension (LRH) is multifactorial and includes infrequent inherited genetic syndromes, milder phenotypes of classic diseases and environmental exposures. All these conditions have in common a higher cardiovascular risk mediated by the over activation of the mineralocorticoid receptor $(M R)$, present not only in the kidney, but also in vasculature, myocardium and adipocytes. Consequently, the aim of LRH treatment goes beyond the control of blood pressure and requires antagonizing $M R$ with specific pharmacologic agents, pursuing normalization of renin as a clinical objective. Due to the unusual evaluation of renin status by non-endocrinologists and lack of disease awareness, only a minority of hypertensive patients receive this pathophysiologically-driven treatment that should reduce cardiovascular outcomes.

(Rev Med Chile 2019; 147: 490-498)

Key words: Aldosterone; Hypertension; Receptors, Mineralocorticoids; Renin; Spironolactone.

\section{Importancia del problema}

L a hipertensión arterial (HTA) es un problema de salud frecuente en nuestro país, según los datos de la Encuesta Nacional de Salud 2016-2017 (ENS 2016-2017), 27,6\% de la población tiene sospecha de la enfermedad ${ }^{1}$. Si consideramos que la hipertensión hiporreninémica se describe hasta en $30 \%$ de los hipertensos ${ }^{2}$, esto representa $\approx 1,5$ millones de pacientes en nuestro país, según los datos del censo $2017^{3}$.

La hipertensión hiporreninémica (HTAH) agrupa a un sinnúmero de condiciones cuyo mecanismo fisiopatológico de elevación de la presión arterial es la activación del receptor mineralocorticoide (MR) con la consiguiente activación del canal epitelial de sodio en el nefrón distal $(\mathrm{ENaC})$, que aumenta directamente la reabsorción de sodio y secundariamente agua por las vías que le son propias. Esto, en definitiva, es censado por la mácula densa, disminuyendo los niveles de renina ${ }^{4,5}$.

No obstante, el receptor de mineralocorticoides no solo se encuentra en el nefrón distal, sino que también en el miocardio, endotelio, colon, cerebro y células dendríticas ${ }^{6}$, produciendo daño cardiovascular independiente del control de la presión arterial. Es así como en un estudio en pacientes con hiperaldosteronismo primario, a igual presión arterial, aquellos que no lograron normalizar renina (mayor activación del MR), tenían mayores desenlaces adversos cardiovasculares, manifestados por fibrilación auricular (FA), infarto agudo al miocardio (IAM)/revascularización coronaria y muerte ${ }^{7}$. Por lo tanto, el bloqueo específico del MR, aparte de normalizar renina y presión arterial, atenúa los efectos deletéreos en otros tejidos. 
Presentamos esta revisión con énfasis clínico, a manera de simplificar su aplicación en el nivel primario y secundario de salud, tanto público como privado.

\section{Introducción: fisiología y fisiopatología}

El sistema renina-angiotensina-aldosterona (SRAA) se desarrolló temprano en la evolución, a manera de mantener la volemia y presión arterial en un ambiente escaso en sodio.

Un estudio clásico mostró que sujetos de la etnia yanomani (con dieta sin sal) tienen un eje SRAA "hiperactivado" en comparación con sujetos con dieta occidental, con niveles de renina y aldosterona significativamente más elevados, pero sorprendentemente con menor presión arterial y escasa natriuresis ${ }^{8}$. Este hallazgo sugiere que nuestro consumo de sodio actual (9,4 g promedio según ENS 2016-2017) $)^{9}$ obliga a nuestro SRAA a suprimirse, y el hallazgo fisiopatológico más frecuente en la actualidad es la producción de aldosterona inadecuada o autónoma para nuestro consumo de sodio.

Con respecto a su regulación, la aldosterona tiene 3 secretagogos principales: angiotensina II e hiperkalemia y, en menor medida, ACTH, regulando tanto la volemia (vía eje renina angiotensina) como la kalemia (vía activación de canal ROMK).

Es así como en presencia de hipovolemia, aumenta renina, angiotensina II y aldosterona, restaurando la volemia, pero limitando la kaliuresis, tanto por el mínimo aporte de sodio en el nefrón distal como el bloqueo de ROMK inducido por angiotensina II. Esto es un hiperaldosteronismo dependiente de renina ("secundario") y considerado fisiológico ${ }^{10}$.

Existen pequeñas diferencias raciales en la regulación del eje. Es así como en hipertensos de raza negra, frente a la depleción de sodio, tienen un menor aumento de renina y una mayor caída de presión arterial ${ }^{11}$. Esto sugiere supresión del SRAA con mayor tendencia a la HTAH.

\section{Definición de la HTA hiporreninémica}

Tal como se señaló anteriormente, el fenotipo de HTAH se define como aquel caracterizado por la sobreactivación del MR, con la consiguiente reabsorción primaria de sodio por canales $\mathrm{ENaC}$ y reabsorción secundaria de agua por las vías que le son propias, lo que suprime renina por hipervolemia. La causa específica de la sobreactivación del MR puede ser dependiente o independiente de aldosterona. Este fenotipo se ha descrito e investigado hace más de 50 años ${ }^{12,13}$, sin embargo, cada vez está cobrando mayor relevancia.

La medición de renina puede realizarse de 2 formas, medición de su concentración plasmática (DRC, por sus siglas en inglés) o de su capacidad para transformar el angiotensinógeno en angiotensina I (PRA, por sus siglas en inglés). Esta última técnica puede realizarse mediante radioinmunoensayo (RIA) (en abandono por el uso de radiactividad) o espectrometría masa-masa (costosa y pobremente estandarizada). La medición de concentración de renina directa (DRC) tiene la ventaja de ser más barata y operador independiente, por lo que es la técnica más utilizada en la actualidad.

Si bien es materia de controversia, se acepta como renina suprimida a: Renina directa (DRC) $<6-10 \mathrm{uUI} / \mathrm{mL}$ o una actividad de renina en plasma $($ ARP $)<0,8-1,0 \mathrm{ng} / \mathrm{mL} / \mathrm{h}$ dependiendo del método utilizado y la ingesta de sodio del paciente $^{5}$. Puede estimarse ARP dividiendo DRC por un factor de $8,2^{14}$. No se han establecido puntos de corte según etnia.

\section{Causas de hipertensión hiporreninémica}

Existen 2 extremos del fenotipo de HTAH según los niveles de aldosterona (Tabla 1). Si esta está elevada, en el contexto de renina suprimida, la única posibilidad diagnóstica es hiperaldosteronismo primario clásico. Esto porque la aldosterona activará MR y suprimirá renina por hipervolemia.

En el otro extremo (aldosterona baja o suprimida) existen múltiples condiciones congénitas y adquiridas. Todas ellas activan el MR, por un mecanismo independiente de aldosterona, lo que suprime renina por hipervolemia. La causa específica de hiperactivación del MR en estas condiciones varía de quien activa $\mathrm{MR}$, pero en general puede ser por deoxicorticosterona (DOC); potente mineralocorticoide precursor en la síntesis de aldosterona como en la hiperplasia suprarrenal congénita por 11 y 17 hidroxilasa, o por cortisol, 
como en el síndrome de exceso aparente de mineralocorticoides (SEAM) o el síndrome de Cushing.

Un punto intermedio lo representa el fenotipo de HTAH con aldosterona normal, ya que aquí se manifiestan condiciones más leves que aún no elevan o no suprimen esta hormona. Ejemplos del primer caso es el hiperaldosteronismo primario leve o moderado y del segundo el SEAM no clásico, tal como se detallará más adelante.

\section{Hiperaldosteronismo primario}

El hiperaldosteronismo primario (HAP) es la causa más frecuente de hipertensión hiporreninémica. Se define desde el punto de vista fisiopatológico como una secreción inapropiada de aldosterona para la volemia y kalemia. Clínicamente se manifiesta como hipertensión arterial de severidad variable, con hipokalemia cada vez en menos porcentaje de los casos ${ }^{10}$. Su espectro se está ampliando, reconociéndose actualmente versiones leves y normotensivas de la enfermedad ${ }^{10,15,16}$.

Desde el punto de vista operacional las guías actuales de la Endocrine Society proponen tamizaje de $\mathrm{HAP}^{14}$ en los siguientes criterios clínicos:

1. $\mathrm{PA}>150 / 100 \mathrm{mmHg}$ en 3 o más ocasiones.

2. $\mathrm{PA}>140 / 90 \mathrm{mmHg}$ resistente a 3 o más fármacos.

3. $\mathrm{PA}<140 / 90 \mathrm{mmHg}$ pero con 4 fármacos.

4. HTA con hipokalemia, hallazgo de incidentaloma suprarrenal o apnea del sueño.

5. Antecedente familiar de HAP, accidente vascular encefálico o HTA en menores de 40 años.

Con respecto a los criterios bioquímicos, está actualmente en desuso un criterio muy estricto como sería usar aldosterona $>15 \mathrm{ng} / \mathrm{dL}$, renina suprimida con relación aldosterona/ARP $>30$. La razón es que estos criterios de tamizaje, tanto clínicos como bioquímicos, están diseñados para casos graves de HAP, por lo tanto, donde el daño cardiovascular ${ }^{17,18,19}$ y renal ${ }^{20,21}$ asociado a la activación del MR ya se ha producido.

Es por ello es que recientemente hemos propuesto criterios menos estrictos para detectar casos más leves y en etapas iniciales que se beneficien de tratamiento precoz, tales como: HTA en paciente joven $(<40$ años), hipokalemia sin HTA, HTA de difícil control o fibrilación auricular aislada con poco daño cardiaco estructural. Consisten- temente, nuestro grupo utiliza puntos de cortes más sensibles, validados por las nuevas guías de $\mathrm{HAP}^{10}$, para evitar falsos negativos, usando como criterio bioquímico una aldosterona $>6-9 \mathrm{ng} / \mathrm{dL}$ en el contexto de renina suprimida.

Con respecto a la medición de aldosterona y renina, destacamos que no es necesario cambiar fármacos previo al estudio, especialmente si el paciente usa más de dos fármacos y el estudio se realiza sin restricción de sal, ya que este puede interpretarse en el contexto clínico. Por ejemplo, un paciente usuario de valsartán/hidroclorotiazida que presenta renina suprimida orienta aun más al diagnóstico de hipertensión hiporreninémica, dado que ambos fármacos suben renina. Sí debe siempre suspenderse espironolactona/eplerenona por al menos 4 semanas. En caso de monoterapia e HTA de buen control se puede cambiar a amlodipino o doxazosina por 4 a 6 semanas, dado su efecto neutro en el SRAA.

Otros confundentes en la medición de renina $\mathrm{y}$ aldosterona (falsos positivos y negativos) se describen en la Tabla 2.

Es fundamental destacar que, anulando todos los posibles confundentes, el test confirmatorio no siempre es necesario. Es así como las nuevas guías de HAP recomiendan no hacerlo si: aldosterona

\section{Tabla 1. Causas de hipertensión hiporreninémica}

\section{Aldosterona no suprimida ( $>6 \mathrm{ng} / \mathrm{dL}$ )}

Hiperaldosteronismo primario clásico

Aldosterona baja $(<6 \mathrm{ng} / \mathrm{dL})$

1. Causas congénitas

a) Síndrome de exceso aparente de mineralocorticoides (SEAM)

b) Hiperplasia suprarrenal congénita por déficit de $11 \beta$ y $17 \alpha$ hidroxilasa

c) Mutación activante ENaC (síndrome de Liddle)

d) Síndrome de Gordon

e) Mutación inactivante del receptor de glucocorticoides

f) Mutación activante del receptor de mineralocorticoides

2. Causas adquiridas

a) Inhibición reversible $11 \beta \mathrm{HSD} 2$ por regaliz o de jugo de pomelo

b) Síndrome de Cushing clínico

c) Consumo excesivo de sodio

$11 \beta \mathrm{HSD} 2$ = 11 beta hidroxiesteroide deshidrogenasa tipo 2 . $\mathrm{ENaC}=$ Epithelial sodium channel. 
Tabla 2. Confundentes en la medición de renina y aldosterona

\begin{tabular}{|ll|}
\hline Confundente & Comentarios \\
Kalemia & La hipokalemia debiera fisiológicamente disminuir aldosterona y viceversa. Renina no \\
se afecta & El SRAA está diseñado para activarse en condiciones de restricción de sal. Es por ello \\
Consumo de sal & que no debe indicarse durante la medición del examen, ya que evitará la detección de \\
& renina suprimida
\end{tabular}

SRAA = Sistema renina-angiotensina-aldosterona. $M R=$ receptor de mineralocorticoides. $\mathrm{iECA}=$ inhibidores de la enzima convertidora de angiotensina. ARA2 = Bloqueadores del receptor de angiotensina 2 . AINES $=$ antiinflamatorios no esteroidales.

$>20 \mathrm{ng} / \mathrm{dL}$ en presencia de renina suprimida, hipertensión e hipokalemia ${ }^{10}$. Por otra parte, un estudio de cohorte mostró $29 \%$ de falsos negativos al realizar el test de sobrecarga salina en posición acostado $^{22}$.

Considerando que la hiperplasia adrenocortical bilateral y el adenoma productor de aldosterona concentra alrededor de $99 \%$ de las causas de hiperaldosteronismo primario ${ }^{23}$, desde el punto de vista práctico es planteable realizar tomografía computada (TAC) de abdomen y, si no hay adenomas suprarrenales de resolución quirúrgica (o no es posible realizar cirugía), tratar empíricamente con antagonistas del MR, con el objetivo de normalizar la presión y renina (Figura 1).

Es importante aclarar que, si bien las guías de la Endocrine Society ${ }^{14}$ siguen recomendando el muestreo de venas suprarrenales para probar lateralización del HAP, en nuestro grupo hemos considerado solo hacerlo en casos de adenomas bilaterales con intención de resolución quirúrgica, considerando la baja disponibilidad/experiencia del procedimiento en nuestro país y el riesgo de hemorragia suprarrenal. Por otro lado, un estudio con distribución aleatoria de la muestra mostró similares tasas de cura bioquímica, comparando TAC de abdomen versus muestreo de venas suprarrenales y que la mayoría de los errores del TAC son en detectar hiperplasias unilaterales y no el lado funcional en presencia de adenoma ${ }^{24}$.

\section{Hipertensión hiporreninémica hipoaldostero- némica}

Cuando un paciente presenta renina baja y aldosterona baja $(<6 \mathrm{ng} / \mathrm{dL})$, se considera una HTA no dependiente de aldosterona y representa un abanico de opciones de baja prevalencia y difícil estudio, por lo que deben ser derivados a centros especializados.

\section{Síndrome de exceso aparente de mineralocorti- coides (SEAM)}

El receptor de mineralocorticoides tiene la misma afinidad por cortisol que por aldosterona y circula en concentraciones 100 a 1.000 veces mayor $^{25,26}$. La enzima $11 \beta$ HSD tipo 2 se encarga 


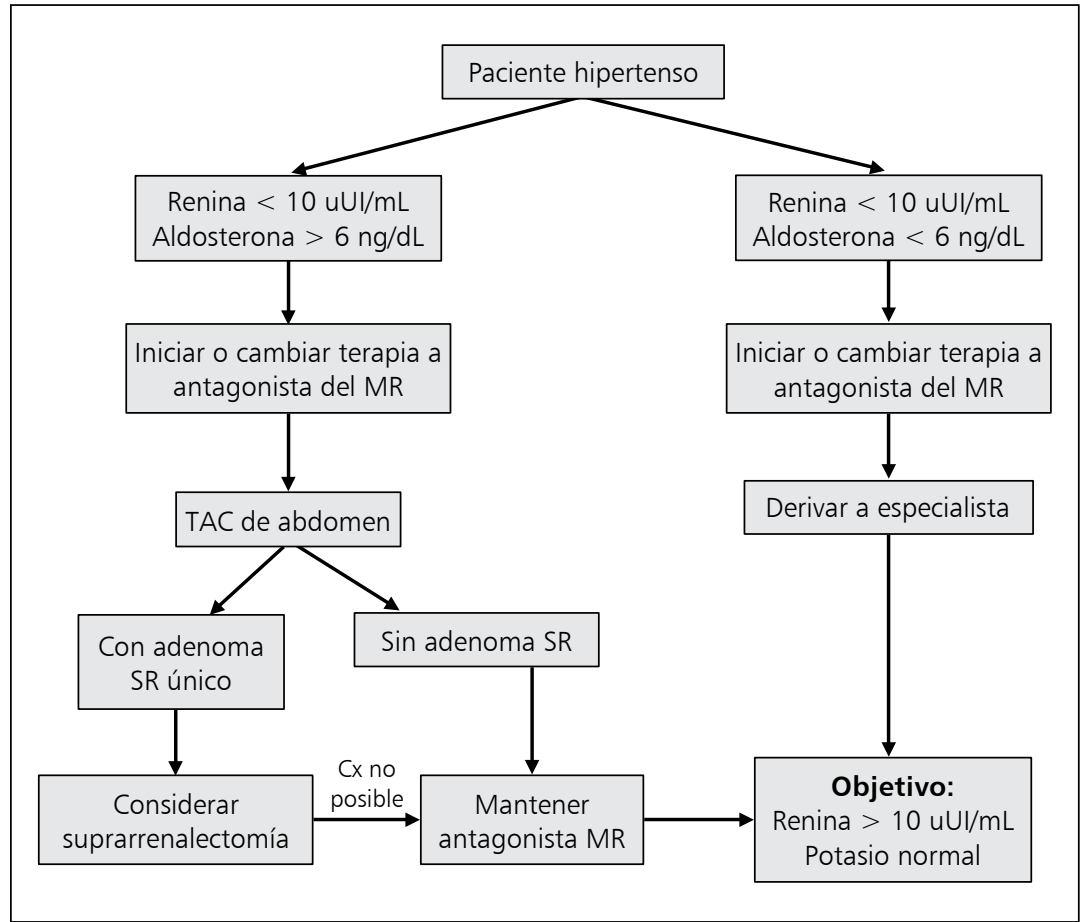

Figura 1. Algoritmo de manejo en pacientes con hipertensión hiporreninémica. Recordar que el estudio puede realizarse bajo terapia antihipertensiva. de inactivar cortisol a cortisona a nivel renal, de manera de evitar la activación del MR. Si esta enzima está mutada o inhibida, se producirá sobreactivación del MR, aumentando la volemia, lo que en consecuencia suprimirá renina, dando origen a hipertensión hiporreninémica. El cuadro clásico se describió en 1979 en dos pacientes con debut de HTA en la edad pediátrica, hipokalemia y elevación de la relación cortisol/cortisona ${ }^{27}$. Sin embargo, su espectro se está expandiendo cada vez más, describiéndose mutaciones parciales con hipertensión hiporreninémica leve ${ }^{28}$, a lo que se le ha denominado SEAM no clásico. El diagnóstico se sospecha con el fenotipo de HTAH y se confirma con la elevación de la relación cortisol/cortisona en suero u orina, con o sin secuenciación de $11 \beta$ HSD tipo 2.

Recientemente, nuestro grupo reclutó 127 pacientes adolescentes y adultos hipertensos de atención primaria en Santiago y a todos se les secuenció el gen de la enzima $11 \beta$ HSD tipo $2^{29}$. Se vio que en 9/127 (7,1\%) tenían elevación de la relación cortisol/cortisona, sugerente de SEAM no clásico. Estos sujetos tenían presión arterial sistólica más elevadas, menores concentraciones de renina y mayor excreción de potasio, sugerentes de mayor activación del MR. En la minoría de los sujetos con sospecha de SEAM no clásico se encontró mutación de la enzima $11 \beta$ HSD tipo 2 . Este hallazgo es muy importante, sugiriendo que un subgrupo con falla parcial no genética de 11HSD2 son subdiagnosticados y se beneficiarían de bloqueo del MR, independiente del control de la PA.

\section{Síndrome de Cushing}

En cuanto al hipercortisolismo, cualquiera sea su causa, el mecanismo principal de génesis de HTAH es la incapacidad de la enzima $11 \beta$ HSD tipo 2 de inactivar el cortisol producido en exceso, por ende, activando excesivamente el MR. Si a esto se le suma tanto el trastorno de los hidratos de carbono (prediabetes y diabetes), como a la obesidad que la condición produce, estamos frente a un paciente de alto riesgo cardiovascular ${ }^{30}$. Frecuentemente se confunde con síndrome metabólico y los hallazgos clínicos específicos que permiten reconocer el síndrome de Cushing son: miopatía 
proximal, fragilidad capilar, estrías rojo vinosas $>1 \mathrm{~cm}$ de ancho y facies pletórica ${ }^{31}$.

Por otra parte, también se ha descrito el síndrome de Cushing subclínico, es decir, pacientes que tienen una alteración bioquímica de hipercortisolismo (test de Nugent $>1,8 \mathrm{ug} / \mathrm{dl}$ ), pero sin los hallazgos clínicos específicos anteriormente mencionados. Frecuentemente, estos pacientes son hipertensos por el mismo mecanismo descrito para el cuadro clínico clásico.

Considerando que en ambas condiciones se produce una sobreactivación del MR, es lógico intentar bloquear la alteración fisiopatológica inicial con antagonistas de este receptor.

\section{Hiperplasia suprarrenal congénita por déficit de $11 \beta$ y $17 \alpha$ hidroxilasa}

El mecanismo fisiopatológico común es una acumulación de deoxicorticosterona (DOC) por el déficit enzimático respectivo a nivel de la esteroidogénesis suprarrenal ${ }^{32}$. El DOC es un potente mineralocorticoide que activará al MR tal como la aldosterona y, por ende, suprimirá compensatoriamente renina. En el caso del déficit de $11 \beta$ hidroxilasa habrá signos de hiperandrogenismo (hirsutismo, acné, oligomenorrea) en la mujer y pubertad precoz en el hombre. Por otra parte, si existe déficit de $17 \alpha$ hidroxilasa se manifestará en casos extremos como fenotipo femenino en sujetos $46 \mathrm{XY}$ o, en casos más leves, con signos de hipogonadismo (ginecomastia, testículos pequeños, ausencia de vello). En la mujer debuta como pubertad retrasada con amenorrea primaria o secundaria.

\section{Síndrome de Liddle}

Ocurre por una mutación activante en el canal epitelial de sodio ENaC. Si bien no hay activación del MR, funciona como tal, porque el mecanismo de producción de HTA en la sobreactivación de MR es la reabsorción de sodio vía $\mathrm{ENaC}$, con reabsorción secundaria de agua. Si bien el cuadro clásico se presenta en pacientes jóvenes con hipokalemia, cada vez se están describiendo mutaciones menos graves. Es así como en pacientes africanos con hipertensión arterial resistente, se encontraron mutaciones en $30 \%$ de ellos ${ }^{33}$. Además, se describe en la literatura el diagnóstico en pacientes adultos, incluso en una paciente octogenaria ${ }^{34}$. La sospecha del cuadro puede hacerse clínicamente, al tener el fenotipo de HTAH, que no responde desde el punto de vista clínico (no disminuye PA) o bioquímico (no normaliza renina) con el uso de antagonistas del MR. En ese caso deben utilizarse antagonistas de $\mathrm{ENaC}$, como amiloride o triamterene.

\section{Manejo clínico de los pacientes con hipertensión arterial hiporreninémica}

Primero que todo debe confirmarse el fenotipo de hipertensión hiporreninémica, ya que implica una condición crónica. Deben minimizarse falsos positivos y negativos ya mencionados. Recordamos que el examen debe realizarse en un laboratorio certificado, con el paciente sentado luego de 30 min de reposo, sin restricción de sal y con medición concomitante de potasio plasmático. En la Figura 1 se resume el algoritmo de manejo propuesto.

En cuanto al HAP, si un paciente se presenta con estudio compatible (renina suprimida y aldosterona elevada) e hipokalemia, no es necesario realizar test confirmatorio. En tal caso, junto con iniciar tratamiento específico, debe procederse a una tomografía computada de abdomen para precisar la causa del HAP. En caso de encontrar adenoma único, no es necesario realizar muestreo de venas suprarrenales y debe plantearse resolución quirúrgica. Un estudio mostró que, luego de suprarrenalectomía, las tasas de curación bioquímica alcanzaron a 95\%; sin embargo, solo $50 \%$ logró normalizar PA, enfatizando que el diagnóstico precoz del HAP evita el daño cardiaco y vascular asociado ${ }^{35}$.

Si por cualquier motivo no puede realizarse el test confirmatorio, sugerimos iniciar tratamiento antihipertensivo con antagonistas del MR. Tal como se mencionó anteriormente, este receptor es ubicuo, por lo que el objetivo del tratamiento no debería ser solo normalizar PA, sino que normalizar renina, idealmente a valores de renina directa $>10 \mathrm{uUI} / \mathrm{mL}$ o de actividad de renina en plasma $>1 \mathrm{ng} / \mathrm{mL} / \mathrm{h}^{10}$. Esto incluso ha sido confirmado por estudios epidemiológicos, en que los pacientes que lograron normalizar renina tenían un curso similar a hipertensos esenciales, sin embargo, 
los que persistían con renina suprimida bajo tratamiento tenían mayor incidencia de eventos cardiovasculares ${ }^{7}$.

En nuestra opinión, la mayoría de los pacientes con hipertensión hiporreninémica debiera recibir antagonistas del MR, ya que corrige la alteración fisiopatológica inicial. Sin embargo, una minoría de ellos estaría recibiendo este tratamiento. Esto ha sido demostrado en el estudio Pathway-2, en que los antagonistas del MR fueron superiores a doxazosina o bisoproplol en HTA resistente, donde los niveles de renina predicen la respuesta ${ }^{36}$. Si el paciente no responde a la terapia y persiste renina suprimida, debe sospecharse síndrome de Liddle e iniciar antagonista del receptor $\mathrm{ENaC}$ como amiloride o triamterene.

En cuanto a las alternativas disponibles, actualmente contamos con espironolactona y eplerenona. Espironolactona, además de antagonizar el MR, bloquea el receptor de andrógenos, lo que limita su uso en hombres por las manifestaciones clínicas de hipogonadismo. Sin embargo, tiene la ventaja de ser de menor costo que eplerenona. Eplerenona es un antagonista específico del MR, la dosis de inicio es $50 \mathrm{mg}$ al día, con un máximo de 200 mg al día. Dentro de los efectos adversos se anotan hiperkalemia, dependiendo de la dosis y función renal del paciente.

Prometedora es la llegada de finerenona, un antagonista no esteroidal del receptor de mineralocorticoides, 10 veces más potente que eplerenona. Tendría menor incidencia de hiperkalemia al tener menor afinidad por el MR renal. Se ha demostrado su utilidad en disminuir albuminuria en nefropatía diabética ${ }^{37}$.

\section{Conclusiones y reflexiones}

La HTAH representa a un subgrupo de hipertensos, con una fisiopatología similar, una alta concentración de causas endocrinas y muy prevalente (hasta 30\%) que, actualmente, está subdiagnosticada y subtratada.

Presentamos un algoritmo de manejo práctico (Figura 1) para simplificar su aplicación en el nivel primario y secundario de salud, tanto público como privado.

Si bien no se han realizado estudios epidemiológicos que demuestren que la medición rutinaria de renina disminuya los costos en salud, en diver- sas publicaciones se propone el uso de renina para guiar la terapia antihipertensiva ${ }^{38}$. En la medida en que se avance en las distintas causas de HTA, la causa esencial o idiopática tenderá a ser cada vez menos prevalente. No es lógico tratar a todos los hipertensos de la misma manera, debemos avanzar hacia una medicina personalizada.

Considerando que la hipertensión hiporreninémica estaría afectando a más de 1 millón de personas que viven en Chile, sugerimos la medición rutinaria de renina a todos los pacientes y su incorporación a las garantías explícitas en salud (GES), lo que sin duda disminuirá los costos, tanto para los pacientes como para el sistema de salud en globo.

\section{Referencias}

1. Encuesta nacional de salud 2016-2017. Disponible en http://www.minsal.cl/wp-content/uploads/2017/11/ ENS-2016-17_PRIMEROS-RESULTADOS.pdf.

2. Mulatero P, Verhovez A, Morello F, Veglio F. Diagnosis and treatment of low-renin hypertension. Clin Endocrinol 2007; 67: 324-34.

3. http://www.censo2017.cl/descargas/home/sintesis-de-resultados-censo2017.pdf.

4. Baudrand R, Vaidya A. The Low-Renin Hypertension Phenotype: Genetics and the Role of the Mineralocorticoid Receptor. Int J Mol Sci 2018; 19 (2). pii: E546.

5. Monticone S, Losano I, Tetti M, Buffolo F, Veglio F, Mulatero, P. Diagnostic approach to low-renin hypertension. Clin Endocrinol (Oxf) 2018; 89 (4): 385-96.

6. Ferrario C, Schiffrin E. Role of Mineralocorticoid Receptor Antagonists in Cardiovascular Disease. Circulation Research 2015; 116 (1): 206-13.

7. Hundemer GL, Curhan GC, Yozamp N, Wang M, Vaidya A. Cardiometabolic outcomes and mortality in medically treated primary aldosteronism: a retrospective cohort study. Lancet Diabetes Endocrinol 2018; 6 (1): 51-9.

8. OliverWJ, Cohen EL, Nell JV. Blood Pressure, Sodium Intake, and Sodium Related Hormones in the Yanomamo Indians, a "No-salt" Culture. Circulation 1975; 52 (1): 146-51.

9. Encuesta nacional de salud 2016-2017. Disponible en http://www.minsal.cl/wp-content/uploads/2018/ 01/2-Resultados-ENS_MINSAL_31_01_2018.pdf.

10. Vaidya A, Mulatero P, Baudrand R, Adler GK. The Expanding Spectrum of Primary Aldosteronism: Implications for Diagnosis, Pathogenesis, and Treatment. Endocr Rev 2018; 39 (6): 1057-88. 
11. He FJ, Markandu ND, Sagnella GA, MacGregor GA. Importance of renin system in determining blood pressure fall with salt-restriction in black and white hypertensives. Hypertension 1998; 32: 820-4.

12. Jose A, Crout JR, Kaplan NM. Suppressed plasma renin activity in essential hypertension. Roles of plasma volume, blood pressure, and sympathetic nervous system. Ann Intern Med 1970; 72 (1): 9-16.

13. Channick BJ, Adlin EV, Marks AD. Suppressed plasma renin activity in hypertension. Arch Intern Med 1969; 123 (2): 131-40.

14. Funder J, Carey R, Mantero F, Murad M, Reincke M, Shibata $\mathrm{H}$, et al. The Management of Primary Aldosteronism: Case Detection, Diagnosis, and Treatment: An Endocrine Society Clinical Practice Guideline. J Clin Endocrinol Metab 2016; 101 (5): 1889-916.

15. Baudrand R, Guarda F, Fardella C, Hundemer G, Brown J, Williams G, et al. Continuum of Renin-Independent Aldosteronism in Normotension. Hypertension 2017; 69 (5): 950-6.

16. Baudrand R, Guarda F, Torrey J, Williams G, Vaidya A. Dietary Sodium Restriction Increases the Risk of Misinterpreting Mild Cases of Primary Aldosteronism. J Clin Endocrinol Metab 2016; 101 (11): 3989-96.

17. Catena C, Colussi G, Nadalini E, Chiuch A, Baroselli S, Lapenna $\mathrm{R}$, et al. Cardiovascular outcomes in patients with primary aldosteronism after treatment. Arch Intern Med 2008; 168 (1): 80-5.

18. Milliez P, Girerd X, Plouin PF, Blacher J, Safar ME, Mourad JJ. Evidence for an increased rate of cardiovascular events in patients with primary aldosteronism. J Am Coll Cardiol 2005; 45 (8): 1243-8.

19. Mulatero P, Monticone S, Bertello C, Viola A, Tizzani $\mathrm{D}$, Iannaccone A et al. Long-term cardio- and cerebrovascular events in patients with primary aldosteronism. J Clin Endocrinol Metab 2013; 98 (12): 4826-33.

20. Rossi GP, Bernini G, Desideri G, Fabris B, Ferri C, Giacchetti $\mathrm{G}$, et al. Renal damage in primary aldosteronism: results of the PAPY Study. Hypertension 2006; 48 (2): 232-8.

21. Reincke M, Rump LC, Quinckler M, Hahner S, Deiderich S, Lorenz R, et al. Risk factors associated with a low glomerular filtration rate in primary aldosteronism. J Clin Endocrinol Metab 2009; 94 (3): 869-75.

22. Cornu E, Steichen O, Nogueira-Silva L, Küpers E, Pagny JY, Grataloup C, et al. Suppression of aldosterone secretion after recumbent saline infusion does not exclude lateralized primary aldosteronism. Hypertension 2016; 68 (4): 989-94.

23. Fardella C, Carvajal C. Hiperaldosteronismo primario y otras causas endocrinas de hipertensión arterial. En:
Arteaga E, Baudrand R, Domínguez JM, Editores, Endocrinología Clínica, Segunda Edición. Santiago, Chile: Editorial Mediterráneo Ltda.; 2018.p. 169-74.

24. Dekkers T, Prejbisz A, Kool LJS, Groenewoud HJMM, Velema M, Spiering $\mathrm{W}$ et al. Adrenal vein sampling versus CT scan to determine treatment in primary aldosteronism: an outcome-based randomised diagnostic trial. L Lancet Diabetes Endocrinol 2016; 4 (9): 739-46.

25. Krozowski ZS, Funder JW. Renal mineralocorticoid receptors and hippocampal corticosterone-binding species have identical intrinsic steroid specificity. Proc Natl Acad Sci USA 1983; 80 (19): 6056-60.

26. Arriza JL, Weinberger C, Cerelli G, Glaser TM, Handelin BL, Housman DE, et al. Cloning of human mineralocorticoid receptor complementary DNA: structural and functional kinship with the glucocorticoid receptor. Science 1987; 237 (4812): 268-75.

27. Ulick S, Levine LS, Gunczler P, Zanconato G, Ramirez LC, Rauh W, et al. A syndrome of apparent mineralocorticoid excess associated with defects in the peripheral metabolism of cortisol. J Clin Endocrinol Metab 1979; 49 (5): 757-64.

28. Wilson RC, Dave-Sharma S, Wei JQ, Obeyesekere VR, Li K, Ferrari P, et al. A genetic defect resulting in mild low-renin hypertension. Proc Natl Acad Sci USA 1998; 95 (17): 10200-5.

29. Tapia-Castillo A, Baudrand R, Vaidya A, Campino C, Allende F, Valdivia C, et al. Clinical, Biochemical and Genetic Characteristics of "Non-Classical" Apparent Mineralocorticoid Excess Syndrome. J Clin Endocrinol Metab 2019; 104 (2): 595-603.

30. Fassnacht M, Arlt W, Bancos I, Dralle H, Newell-Price J, Sahdev A, et al. Management of adrenal incidentalomas: European society of endocrinology clinical practice guideline in collaboration with the european network for the study of adrenal tumors. Eur. J. Endocrinol 2016; 175 (2): G1-G34.

31. Carrasco C, Baudrand R. Síndrome de Cushing. En: Arteaga E, Baudrand R, Domínguez JM, Editores, Endocrinología Clínica, Segunda Edición. Santiago, Chile: Editorial Mediterráneo Ltda.; 2018. p. 148-55.

32. Khattab A, Haider S, Kumar A, Dhawan S, Alam D, Romero R et al.. Clinical, genetic, and structural basis of congenital adrenal hyperplasia due to 11-hydroxylase deficiency. Proc Natl Acad Sci USA 2017; 114 (10): E1933-40.

33. Jones ES, Spence JD, Mcintyre AD, Nondi J, Gogo K, Akintunde A, et al. High frequency of variants of candidate genes in black africans with low renin-resistant hypertension. Am. J. Hypertens 2017; 30 (5): 478-83.

34. Pepersack T, Allegre S, Jeunemaître X, Leeman M, Praet 
JP. Liddle syndrome phenotype in an octogenarian. J Clin. Hypertens 2015; 17 (1): 59-60.

35. Williams TA, Lenders JWM, Mulatero P, Burrello J, Rottenkolber M, Adolf C, et al. Outcomes after adrenalectomy for unilateral primary aldosteronism: an international consensus on outcome measures and analysis of remission rates in an international cohort. Lancet Diabetes Endocrinol 2017; 5 (9): 689-99.

36. Williams B, MacDonald TM, Morant S, Webb DJ, Sever $\mathrm{P}$, McInnes G, et al. Spironolactone versus placebo, bisoprolol, and doxazosin to determine the optimal treatment for drug-resistant hypertension (pathway-2): A randomised, double-blind, crossover trial. Lancet 2015; 386 (10008): 2059-68.

37. Bakris GL, Agarwal R, Chan JC, Cooper ME, Gansevoort RT, Haller H, et al. Effect of Finerenone on Albuminuria in Patients With Diabetic Nephropathy: A Randomized Clinical Trial. JAMA 2015; 314 (9): 884-94.

38. Furberg C. Renin-Guided Treatment of Hypertension: Time for Action. Am J Hypertens 2010; 23 (9): 929-30. 